Stelios F. Assimakopoulos, Georgios K. Markantes, Dimitris Papageorgiou, Irene Mamali, Kostas B. Markou, Markos Marangos and Marina A. Michalaki*

\title{
Low serum TSH in the acute phase of COVID-19 pneumonia: thyrotoxicosis or a face of "non- thyroidal illness syndrome"?
}

https://doi.org/10.1515/cclm-2021-0511

Received April 28, 2021; accepted May 31, 2021;

published online July 12, 2021

Keywords: COVID-19; non-thyroidal illness; thyroid function; thyroid stimulating hormone (TSH); thyrotoxicosis.

To the Editor,

Since the emergence of the coronavirus disease (COVID-19) pandemic, several studies have examined the effects of SARS-CoV-2 infection on thyroid function [1-6]. Available evidence suggests that thyroid function tests (TFT) are altered during COVID-19, but the involved pathophysiological mechanisms have not been clarified. Some investigators have attributed the observed alterations to thyrotoxicosis [1, 2], while others consider them compatible with the "Non-Thyroidal Illness Syndrome" (NTIS) [3-6]. The term NTIS describes the alterations in TFT (namely decreased triiodothyronine-T3, low/normal thyroxine-T4 and thyrotropin-thyroid stimulating hormone (TSH) and increased reverse triiodothyronine-rT3) observed during illness or starvation in individuals without pre-existing thyroid disease and intact hypothalamus-pituitary-thyroid

\footnotetext{
*Corresponding author: Marina A. Michalaki, PhD, Division of Endocrinology - Department of Internal Medicine, University of Patras School of Health Sciences, University Hospital of Patras, Patras, Greece, Phone: +30 2610999427, E-mail: mixmar@upatras.gr. https://orcid.org/0000-0003-2073-8523

Stelios F. Assimakopoulos and Markos Marangos, Department of Internal Medicine, University of Patras School of Health Sciences, Patras, Greece; Division of Infectious Diseases, University of Patras School of Health Sciences, Patras, Greece. https://orcid.org/00000002-6901-3681 (S.F. Assimakopoulos)

Georgios K. Markantes, Irene Mamali and Kostas B. Markou, Division of Endocrinology - Department of Internal Medicine, University of Patras School of Health Sciences, Patras, Greece

Dimitris Papageorgiou, Department of Internal Medicine, University of Patras School of Health Sciences, Patras, Greece
}

(HPT) axis. Our aim was to investigate the alterations in TFT in patients hospitalized for COVID-19 pneumonia within the first $24 \mathrm{~h}$ of admission and before the administration of any medication known to influence the HPT axis, and to examine if such alterations had prognostic significance.

We prospectively studied 22 consecutive patients hospitalized at the University Hospital of Patras from March 3 to May 10, 2020 diagnosed with COVID-19 pneumonia according to established criteria [7]. SARS-CoV-2 infection was confirmed by RT-PCR of nasopharyngeal swab samples. Pneumonia severity classification was based on the American Thoracic Society guidelines [8]. Patients were followed for 14 days for development of severe respiratory failure (SRF) (defined as $\mathrm{PO}_{2} / \mathrm{FiO}_{2}<200$ ) requiring mechanical ventilation (MV). Nineteen age and sex-matched hospitalized patients with community acquired non-COVID-19 pneumonia of the same severity and nineteen healthy persons were included as controls. None of the participants had a history of thyroid disease or was on drugs affecting thyroid function at enrolment. None of our COVID-19 patients received glucocorticoids, dopamine/dobutamine or iodinated contrasts before blood sampling for thyroid function tests. Prophylactic lowmolecular-weight heparin (LMWH) was administered according to COVID-19 treatment guidelines [7], after blood sampling for TFT. The study was approved by the University Hospital of Patras Ethics Committee, while all participants provided informed consent. TSH, total and free thyroxine (T4 and FT4) and triiodothyronine (T3 and FT3), rT3, anti-thyroid peroxidase antibodies (Ab-TPO), CRP, ferritin and D-dimers were measured within $24 \mathrm{~h}$ of admission. Serum T3, FT3, T4, FT4, TSH and Ab-TPO were measured by electrochemiluminescence immunoassays (Cobas e601, Roche Diagnostics ${ }^{\circledR}$, Germany), while rT3 was measured by competitive enzyme immunoassay (BioVendor ${ }^{\circledR}$, Czech Republic).

Data were analyzed using SPSS 25.0 for Windows (SPSS Inc., Chicago, IL). Parameters were tested for 
normality with the Shapiro-Wilk test. Comparisons between groups were performed using Student's $t$-test or oneway ANOVA (normally distributed data) and Mann-Whitney U test or Kruskal-Wallis test (non-normally distributed data). Frequencies were compared with the chi-squared test. Correlations were estimated by Pearson or Spearman correlation tests. All tests were 2-tailed and a p-value of less than 0.05 was considered significant.

Inflammatory markers and D-Dimers were significantly higher in patients with COVID-19 and non-COVID-19 pneumonia compared to healthy controls but did not differ between COVID-19 and non-COVID-19 pneumonia (Table 1). T3, FT3 and TSH were lower, T4 similar, and FT4 and Ab-TPO higher in both patient groups compared to healthy controls. rT3 was slightly higher in COVID-19 patients than in healthy participants (Table 1). Seven COVID-19 patients (31.8\%) had a constellation of low TSH ( $<0.5 \mathrm{mIU} / \mathrm{L}$ ), low/low-normal T3 and FT3, normal T4 and FT4, and increased rT3 (Supplementary Table 1). This pattern was observed in only one patient with nonCOVID-19 pneumonia. Ab-TPO levels were positively correlated with ferritin values in subjects with COVID-19 pneumonia (Spearman's rho $=0.531, \mathrm{p}<0.05$ ).

Higher proportion of males and higher levels of ferritin and/or CRP characterized the COVID-19 patients with severe pneumonia and need for MV. Patients in need for MV had higher Ab-TPO levels, while Ab-TPO positivity was significantly more common in the COVID-19 groups with severe pneumonia and need for MV (Table 2).

In most studies investigating thyroid function in COVID-19, including ours, TSH levels are decreased in COVID-19 patients. However, the interpretations of this finding are discordant. Lania et al. [1] reported thyrotoxicosis based on low serum TSH in $20.2 \%$ of their 287 COVID-19 patients, but only half of them had elevated FT4 or FT3. Moreover, $51.7 \%$ of the thyrotoxic patients had received LMWH prior to thyroid hormone measurements. It is known that LMWH administration artefactually increases serum FT4 up to 5-fold (an in vitro effect due to a significant increase of non-esterified fatty acids, which compete with thyroid hormones for binding to the thyroxine binding globulin) [9]. Similar findings were reported by Muller et al. [2]. These studies postulated the existence of a silent destructive thyroiditis either due to cytokine storm or to a direct effect of the virus on the thyroid gland. Indeed, ACE2, the receptor used by SARS-CoV-2 to enter the cells is expressed in thyroidal follicular cells, but post-mortem studies of COVID-19 patients revealed no significant abnormalities in thyroid morphology and failed to detect SARS-CoV-2 in thyroid tissue [10, 11]. Other studies [3-6] consider the NTIS a more plausible explanation for the altered TFT in COVID-19. Distinguishing between thyrotoxicosis and NTIS may be difficult in hospitalized patients. Overt thyrotoxicosis is typically characterized by TSH $<0.01 \mathrm{mIU} / \mathrm{L}$ (when a sensitive TSH assay is used) [12] and elevated thyroid hormones, while in subclinical hyperthyroidism TSH suppression is less pronounced $(<0.5 \mathrm{mIU} / \mathrm{L})$ and thyroid hormone levels are high-normal. None of our patients had TSH $<0.01 \mathrm{mIU} / \mathrm{L}$ or elevated/high-normal T3, FT3 or T4, making overt thyrotoxicosis less likely. Instead, our COVID-19 patients had alterations in TFT that were similar to those observed in non-COVID-19 pneumonia subjects and mostly consistent with NTIS. Besides, rT3 was $\cong 12.5 \%$ higher in our COVID-19

Table 1: Inflammatory markers and thyroid function tests in healthy controls and in patients with COVID-19 and non-COVID-19 pneumonia, measured within $24 \mathrm{~h}$ of admission.

\begin{tabular}{|c|c|c|c|}
\hline Parameters at admission (normal range) & Healthy controls $(n=19)$ & $\begin{array}{r}\text { Community-acquired non-COVID-19 } \\
\text { pneumonia }(n=19)\end{array}$ & COVID-19 pneumonia $(n=22)$ \\
\hline Gender & $9 \mathrm{M} / 10 \mathrm{~F}$ & $9 \mathrm{M} / 10 \mathrm{~F}$ & $11 \mathrm{M} / 11 \mathrm{~F}$ \\
\hline Age, years & $59(55-64)$ & $63(55-67)$ & $63(55-67)$ \\
\hline $\mathrm{CRP}(<5 \mathrm{mg} / \mathrm{L})$ & $2.0(1.0-3.0)$ & $79.0(29.4-158.0)^{c}$ & $48.4(28.4-145.1)^{c}$ \\
\hline Ferritin $(16,323 \mu \mathrm{g} / \mathrm{L})$ & $69(46-95)$ & $243(170-1,627)^{c}$ & $328(181-1,574)^{c}$ \\
\hline D-dimers $(<2.74 \mathrm{nmol} / \mathrm{L})$ & $0.0(0.0-0.55)$ & $7.23(4.0-21.52)^{c}$ & $9.25(4.60-15.0)^{c}$ \\
\hline T3 $(1.23,3.08 \mathrm{nmol} / \mathrm{L})$ & $1.66 \pm 0.23$ & $1.17 \pm 0.231^{c}$ & $1.40 \pm 0.45^{\mathrm{a}}$ \\
\hline FT3 $(3.1,6.8 \mathrm{pmol} / \mathrm{L})$ & $4.78 \pm 0.54$ & $3.48 \pm 0.88^{c}$ & $3.57 \pm 0.97^{c}$ \\
\hline T4 $(65.64,181.48 \mathrm{nmol} / \mathrm{L})$ & $102.84 \pm 14.42$ & $98.85 \pm 16.09$ & $111.08 \pm 28.70$ \\
\hline FT4 $(11.97,21.88 \mathrm{pmol} / \mathrm{L})$ & $14.42 \pm 1.80$ & $16.86 \pm 3.22^{b}$ & $18.66 \pm 4.38^{c}$ \\
\hline $\mathrm{TSH}(0.27,4.20 \mathrm{mIU} / \mathrm{L})$ & $1.51(1.25-2.44)$ & $1.12(0.80-1.59)^{\mathrm{a}}$ & $1.04(0.39-1.79)^{\mathrm{a}}$ \\
\hline rT3 $(0.11,0.40 \mathrm{nmol} / \mathrm{L})$ & $0.37 \pm 0.08$ & $0.41 \pm 0.09$ & $0.41 \pm 0.06^{\mathrm{a}}$ \\
\hline Ab-TPO (<34 IU/mL) & $10.10(9.10-12.20)$ & $20.60(13.10-28.00)^{b}$ & $18.30(12.95-34.70)^{b}$ \\
\hline
\end{tabular}

M, males; F, females; IU, international units. Data are presented as mean \pm SD (normally distributed) and as median (IQR) (non-normally distributed). ${ }^{a} p<0.05$ vs. healthy controls, ${ }^{b} p<0.01$ vs. healthy controls, ${ }^{c} p<0.001$ vs. healthy controls. 
Table 2: Comparisons of inflammatory markers and thyroid function tests measured within $24 \mathrm{~h}$ of admission, in patients with COVID-19 pneumonia according to disease severity on admission, and according to development of severe respiratory failure requiring mechanical ventilation (MV). Data are presented as mean \pm SD (normally distributed) and as median (IQR) (non-normally distributed).

\begin{tabular}{|c|c|c|c|c|c|c|}
\hline $\begin{array}{l}\text { Parameters at admission } \\
\text { (normal range) }\end{array}$ & $\begin{array}{r}\text { COVID-19 pneumonia } \\
\text { non-severe }(n=13)\end{array}$ & $\begin{array}{r}\text { COVID-19 } \\
\text { pneumonia } \\
\text { severe }(n=9)\end{array}$ & p-Value & $\begin{array}{l}\text { No need for } \\
M V(n=15)\end{array}$ & $\begin{array}{l}\text { Need for } \\
M V(n=7)\end{array}$ & p-Value \\
\hline Gender & $4 \mathrm{M} / 9 \mathrm{~F}$ & $7 \mathrm{M} / 2 \mathrm{~F}$ & 0.030 & $5 \mathrm{M} / 10 \mathrm{~F}$ & $6 \mathrm{M} / 1 \mathrm{~F}$ & 0.022 \\
\hline Age, years & $64(56-67)$ & $62(47-72)$ & NS & $66(56-68)$ & $62(47-76)$ & NS \\
\hline $\operatorname{CRP}(<5 \mathrm{mg} / \mathrm{L})$ & $31.7(21.6-53.0)$ & $128.0(59.0-209.5)$ & 0.012 & $\begin{array}{r}37.8 \\
(24.0-106.8)\end{array}$ & $\begin{array}{r}106.8 \\
(32.3-207.7)\end{array}$ & NS \\
\hline Ferritin $(16,323 \mu \mathrm{g} / \mathrm{L})$ & $186(138-252)$ & $1,416(403-2,277)$ & 0.006 & $224(169-381)$ & $\begin{array}{r}1988 \\
(696-2,695)\end{array}$ & 0.003 \\
\hline D-dimers $(<2.74 \mathrm{nmol} / \mathrm{L})$ & $8.21(4.55-13.03)$ & $14.02(5.80-30.88)$ & NS & $\begin{array}{r}7.83 \\
(4.38-14.89)\end{array}$ & $\begin{array}{r}14.13 \\
(6.41-23.05)\end{array}$ & NS \\
\hline T3 $(1.23,3.08 \mathrm{nmol} / \mathrm{L})$ & $1.48 \pm 0.31$ & $1.31 \pm 0.62$ & NS & $1.48 \pm 0.39$ & $1.25 \pm 0.62$ & NS \\
\hline FT3 $(3.1,6.8 \mathrm{pmol} / \mathrm{L})$ & $3.75 \pm 0.69$ & $3.30 \pm 1.28$ & NS & $3.69 \pm 0.79$ & $3.28 \pm 1.43$ & NS \\
\hline T4 $(65.64,181.48 \mathrm{nmol} / \mathrm{L})$ & $117.90 \pm 25.87$ & $101.17 \pm 31.15$ & NS & $114.68 \pm 26.00$ & $99.24 \pm 36.04$ & NS \\
\hline FT4 $(11.97,21.88 \mathrm{pmol} / / \mathrm{L})$ & $18.92 \pm 3.48$ & $18.28 \pm 5.41$ & NS & $18.66 \pm 3.35$ & $18.53 \pm 6.69$ & NS \\
\hline $\mathrm{TSH}(0.27,4.20 \mathrm{mIU} / \mathrm{L})$ & $0.87(0.45-1.57)$ & $1.37(0.07-2.20)$ & NS & $\begin{array}{r}1.01 \\
(0.45-1.66)\end{array}$ & $\begin{array}{r}1.54 \\
(0.06-2.07)\end{array}$ & NS \\
\hline rT3 $(0.11,0.40 \mathrm{nmol} / \mathrm{L})$ & $0.41 \pm 0.06$ & $0.40 \pm 0.08$ & NS & $0.41 \pm 0.06$ & $0.41 \pm 0.08$ & NS \\
\hline Ab-TPO (<34 IU/mL) & $17.45(11.90-22.45)$ & $31.80(13.85-61.20)$ & NS & $\begin{array}{r}16.90 \\
(12.10-23.30)\end{array}$ & $\begin{array}{r}46.87 \\
(17.30-84.55)\end{array}$ & 0.029 \\
\hline Ab-TPO positive & $7.7 \%$ & $44.4 \%$ & 0.038 & $13.3 \%$ & $50 \%$ & 0.013 \\
\hline
\end{tabular}

M, males; F, females; IU, international units; NS, not significant; $p$-values in bold are those below the set level of significance.

patients compared to healthy controls, a finding typical of NTIS $[12,13]$.

In NTIS, serum T3 typically drops rapidly within $1 \mathrm{~h}$ of the onset of illness and the magnitude of its drop is proportional to disease severity, whereas rT3 increases a few hours later [13]. If the illness is severe or prolonged enough, T4 and TSH also decrease, and a type of transient acquired central hypothyroidism occurs [12]. 31.8\% of our COVID-19 patients had subnormal TSH but normal or even highnormal T4 and FT4. We suggest that this common $[1-4,6]$ pattern in TFT, which could falsely lead to the diagnosis of subclinical thyrotoxicosis, is probably a unique presentation of NTIS in COVID-19. The isolated subnormal serum TSH could be explained by the excessive release of proinflammatory cytokines characterizing COVID-19, as cytokines decrease TRH and TSH secretion [14]. Another potential mechanism might be a direct suppressing effect of SARS-CoV-2 to the hypothalamus and/or pituitary, something which has been shown for SARS-CoV-1 $[15,16]$. Besides, glucocorticoids which are commonly used in COVID patients also suppress TSH. Notably, none of our patients received dexamethasone before blood sampling.

The only thyroid parameter with a prognostic significance in our study was Ab-TPO. Ab-TPO were higher in patients with COVID-19 progressing to SRF and were positively correlated with ferritin, a marker with established prognostic role [17]. Furthermore, Ab-TPO positivity was associated with severe COVID-19 pneumonia and with progression to SRF. The presence of increased autoantibodies other than Ab-TPO has been reported in up to $45 \%$ of COVID-19 patients, and it has been correlated with adverse prognosis [18]. Although the pre-COVID-19 Ab-TPO levels of our patients are unknown, none of them had known rheumatologic/autoimmune diseases and, therefore, it seems that SARS-CoV-2 might trigger thyroid autoimmunity in the context of a generalized immune response, as recently shown [19]. In this framework, Ab-TPO positivity could identify COVID-19 patients with exaggerated immune system activation, being at increased risk of severe/complicated illness. Still, the possibility of SARS-CoV2-induced direct thyroid damage cannot be completely excluded, as destructive thyroiditis could lead to a transient anti-thyroid antibody rise and might also worsen the clinical course of COVID-19 patients. Unfortunately, data regarding longitudinal evolution of Ab-TPO levels in our patients are not available to support this hypothesis.

Our study is limited by the relatively small number of participants and the lack of follow-up of the thyroid function parameters in our patients; thus, more studies are needed in order to confirm our data. 
In conclusion, we have shown that alterations in TFT in glucocorticoid-naive patients with COVID-19 pneumonia are more consistent with NTIS than with thyrotoxicosis. The frequently observed pattern of subnormal TSH, lownormal T3/FT3, normal T4, and high-normal FT4 might represent a unique type of NTIS, manifesting during the acute phase of COVID-19 pneumonia. Finally, positive AbTPO might be a potential adverse prognostic factor.

Research funding: None declared.

Author contributions: Stelios F. Assimakopoulos: conceptualization, writing and critically revising the manuscript. Georgios K. Markantes: statistical analysis, writing the manuscript. Dimitris Papageorgiou: data collection. Irene Mamali: hormonal measurements. Kostas B. Markou: conceptualization, writing and critically revising the manuscript. Markos Marangos: conceptualization, writing and critically revising the manuscript. Marina A. Michalaki: conceptualization, writing and critically revising the manuscript.

Competing interests: Authors state no conflict of interest. Informed consent: Informed consent was obtained from all individuals included in this study.

Ethical approval: Research involving human subjects complied with all relevant national regulations, institutional policies and is in accordance with the tenets of the Helsinki Declaration (as revised in 2013), and has been approved by the authors' Institutional Review Board (University Hospital of Patras Ethics Committee, 9632/17-05-2016).

\section{References}

1. Lania A, Sandri MT, Cellini M, Mirani M, Lavezzi E, Mazziotti G. Thyrotoxicosis in patients with COVID-19: the THYRCOV study. Eur J Endocrinol 2020;183:381-7.

2. Muller I, Cannavaro D, Dazzi D, Covelli D, Mantovani G, Muscatello A, et al. SARS-CoV-2-related atypical thyroiditis. Lancet Diabetes Endocrinol 2020;8:739-41.

3. Chen M, Zhou W, Xu W. Thyroid function analysis in 50 patients with COVID-19: a retrospective study. Thyroid 2021;31:8-11.

4. Khoo B, Tan T, Clarke SA, Mills EG, Patel B, Modi M, et al. Thyroid function before, during, and after COVID-19. J Clin Endocrinol Metab 2021;106:e803-11.

5. Zou R, Wu C, Zhang S, Wang G, Zhang Q, Yu B, et al. Euthyroid sick syndrome in patients with COVID-19. Front Endocrinol 2020;11:566439.

6. Lui DTW, Lee CH, Chow WS, Lee ACH, Tam AR, Fong CHY, et al. Thyroid dysfunction in relation to immune profile, disease status, and outcome in 191 patients with COVID-19. J Clin Endocrinol Metab 2021;106:e926-35.

7. COVID-19 Treatment Guidelines Panel. Coronavirus Disease 2019 (COVID-19) Treatment Guidelines. National Institutes of Health. Available from: https://www.covid19treatmentguidelines.nih. gov/ [Accessed 08 Jan 2020].

8. Metlay JP, Waterer GW, Long AC, Anzueto A, Brozek J, Crothers $\mathrm{K}$, et al. Diagnosis and treatment of adults with community-acquired pneumonia. An official clinical practice guideline of the American thoracic society and infectious diseases society of America. Am J Respir Crit Care Med 2019; 200:e45-67.

9. Mendel CM, Frost PH, Kunitake ST, Cavalieri RR. Mechanism of the heparin-induced increase in the concentration of free thyroxine in plasma. J Clin Endocrinol Metab 1987;65:1259-64.

10. Bradley BT, Maioli H, Johnston R, Chaudhry I, Fink SL, Xu H, et al. Histopathology and ultrastructural findings of fatal COVID-19 infections in Washington state: a case series. Lancet 2020;396: 320-32.

11. Barton LM, Duval EJ, Stroberg E, Ghosh S, Mukhopadhyay S. COVID-19 autopsies, Oklahoma, USA. Am J Clin Pathol 2020;153: 725-33.

12. Fliers $E$, Boelen $A$. An update on non-thyroidal illness syndrome. J Endocrinol Invest 2020, https://doi.org/10.1007/s40618-02001482-4.

13. Michalaki M, Vagenakis AG, Makri M, Kalfarentzos F, Kyriazopoulou V. Dissociation of the early decline in serum T(3) concentration and serum IL- 6 rise and TNF alpha in nonthyroidal illness syndrome induced by abdominal surgery. J Clin Endocrinol Metab 2001;86:4198-205.

14. Croce L, Gangemi D, Ancona G, Liboà F, Bendotti G, Minelli L, et al. The cytokine storm and thyroid hormone changes in COVID-19. J Endocrinol Invest 2021;9:1-14.

15. Wei L, Sun S, Zhang J, Zhu H, Xu Y, Ma Q, et al. Endocrine cells of the adenohypophysis in severe acute respiratory syndrome (SARS). Biochem Cell Biol 2010;88:723-30.

16. Leow MK, Kwek DS, Ng AW, Ong KC, Kaw GJ, Lee LS. Hypocortisolism in survivors of severe acute respiratory syndrome (SARS). Clin Endocrinol 2005;63:197-202.

17. Huang I, Pranata R, Lim MA, Oehadian A, Alisjahbana B. C-reactive protein, procalcitonin, D-dimer, and ferritin in severe coronavirus disease-2019: a meta-analysis. Ther Adv Respir Dis 2020;14: 1753466620937175.

18. Liu Y, Sawalha AH, Lu Q. COVID-19 and autoimmune diseases. Curr Opin Rheumatol 2021;33:155-62.

19. Anaya JM, Monsalve DM, Rojas M, Rodríguez Y, Montoya-García N, Mancera-Navarro LM, et al. Latent rheumatic, thyroid and phospholipid autoimmunity in hospitalized patients with COVID-19. J Transl Autoimmun 2021;4:100091.

Supplementary Material: The online version of this article offers supplementary material (https://doi.org/10.1515/cclm-2021-0511). 